\title{
Multi-objective Optimization of Vertical Profile Trajectory for Civil Aircraft
}

\author{
Suxiao Wang ${ }^{1, *}$, Wei $\mathrm{Shi}^{2}$, and Yingjie $\mathrm{Liao}^{3}$ \\ ${ }^{1}$ Shanghai Aircraft Design and Research Institute, Shanghai, 201210, China \\ ${ }^{2}$ Shanghai INSKY Intelligent Technology Ltd. Co., Shanghai, 201204, China \\ ${ }^{3}$ School of Mechatronic Engineering and Automation, Shanghai University, Shanghai, 200072, China
}

\begin{abstract}
In order to further promote the sustainable development of civil aviation transportation industry, how to reduce the operating cost of civil aircraft has always been the focus of research. This paper establishes a performance optimization model of the aircraft on the vertical flight profile, which takes flight fuel cost and time cost as multi-objective functions and adopts energy state approximation method and optimal control theory. The trajectory of an aircraft in climbing, cruising and descent phases has been optimized using the established model and then simulated with the MATLAB software. The comparison results show that both fuel cost and time cost are significantly reduced through trajectory optimization.
\end{abstract}

\section{Introduction}

In recent years, the increasing aviation flight consumes a large amount of fuel, which is a non-renewable energy. The civil aviation industry is facing the dual pressure of rapid development and reducing energy consumption. Saving energy and reducing fuel consumption is a focus of the civil aviation industry. The least time is of obvious significance to fast-paced modern society. It is the goal of most airlines to complete flight missions in the fastest time. The shortening of time also makes the journey of passengers faster and more convenient.

The main purpose of vertical profile optimization is to reduce fuel consumption or shorten flight time, thereby reducing flight operating costs. In the research on vertical flight profile, A. Franco and D. Rivas use indirect method to solve the four-dimensional trajectory optimization problem with optimal fuel consumption [1]. M. Soler et al. consider aircraft weight, performance, and external environmental factors to achieve four-dimensional trajectory optimization with optimal fuel consumption [2]. S. G. Park et al. use indirect method to optimize the trajectory with the shortest time and the most fuel-efficient performance indicators [3]. S. G. Park and J. B. Clarke use optimal control to determine vertical descent trajectory [4]. O. S. Meric et al. evaluate the vertical descent profile of the aircraft [5]. K. N. Lv and Y. Nan use adaptive genetic algorithm to optimize the trajectory of civil aviation aircraft during the climbing phase with time and fuel optimization as the goal [6].

In summary, most of the current researches focus on a certain phase of vertical flight profile with singleobjective optimization based on fuel consumption or flight time. This paper comprehensively considers flight fuel cost and time cost, takes time and fuel saving as the comprehensive performance optimization goal, and optimizes the flight trajectory of the aircraft in climbing, cruising, and descent phases.

\section{Multi-objective optimization model}

\subsection{Optimization of energy state algorithm}

The energy state method, also known as the energy approximation method, refers to the use of an energy state concept to simplify the aircraft motion model. The energy state model of aircraft particle motion is

$$
\left\{\begin{array}{l}
d E / d t=V(\mathrm{~T}-\mathrm{D}) / \mathrm{W} \\
d x / d t=V+V_{w}
\end{array}\right.
$$

Where, $V_{W}$ is wind speed, $D$ is drag, $\mathrm{W}$ is gravitational force, the state variables are energy $\mathrm{E}$ and distance $\mathrm{x}$, and the control variables are velocity or speed $\mathrm{V}$ and thrust $\mathrm{T}$.

$$
\frac{d x}{d E}=\frac{V+V_{w}}{V(\mathrm{~T}-\mathrm{D}) / \mathrm{W}}
$$

It is believed that the aircraft maintains a fixed altitude and velocity in cruising phase, so the energy of the cruising phase is constant ( $\dot{E}=0)$. The energy of the climbing phase increases monotonically $(\dot{E}>0)$, and the energy of the descent phase decreases monotonously ( $\dot{E}<0$ ). In this way, the energy change is decomposed into the sum of the energy change of the ascending distance $x_{u p}$ and the descending distance $x_{d n}$, then there is

* Corresponding author: wangsuxiao@comac.cc 


$$
\begin{aligned}
\frac{d x}{d E} & =\frac{d\left(x_{u p}+x_{d n}\right)}{d E}=\left.\frac{d x_{u p}}{d E}\right|_{\dot{E}>0}+\left.\frac{d x_{u p}}{d E}\right|_{\dot{E}<0} \\
& =\left.\frac{V_{u p}+V_{\text {Wup }}}{\dot{E}}\right|_{\dot{E}>0}+\left.\frac{V_{d n}+V_{W u p}}{|\dot{E}|}\right|_{\dot{E}<0}
\end{aligned}
$$

In the formula, $V_{u p}$ and $V_{\text {Wup }}$ are airspeed and wind speed in the climbing phase, $V_{d n}$ and $V_{W u p}$ are airspeed and wind speed in the descent phase, respectively.

Equation (3) is the state equation finally used in the trajectory optimization calculation. Energy $E$ is an independent variable and distance $x$ is a state variable.

\subsection{Performance optimization function}

Define the direct operation cost (DOC) of the flight, which comprehensively reflects the cost of fuel and time consumption. It can be written as the following integral form

$$
J=\int_{t_{i}}^{t_{f}}\left(C_{f} \dot{f}+C_{t}\right) d t
$$

Where, $C_{f}$ and $C_{t}$ are the cost per unit mass of fuel and the cost per unit time, respectively, and $\dot{f}$ is the fuel consumption rate. The cost per unit time $C_{t}$ includes all flight costs except fuel, such as labor costs, maintenance costs, depreciation costs, etc. Therefore, the direct Operation cost is a more comprehensive economic indicator.

According to the climbing, cruising, and descent phases of the flight trajectory, it can be rewritten as

$$
\begin{aligned}
J= & \int_{t_{i}}^{t_{t_{i}}}\left(C_{f} \dot{f}+C_{t}\right) d t+\left(R-d_{u p}-d_{d n}\right) \cdot \lambda \\
& +\int_{t_{c f}}^{t_{f}}\left(C_{f} \dot{f}+C_{t}\right) d t
\end{aligned}
$$

The first term of the above formula is the total cost of climbing phase, the second term is the total cost of cruising phase, and the third term is the total cost of descent phase. Where, $\lambda$ is the cost per unit distance in the cruising flight, $\mathrm{R}$ is the flight range of the aircraft, that is, the distance over the ground, $d_{u p}$ and $d_{d n}$ are the ground distances of the climbing phase and descent phase, respectively, $t_{c i}$ is the beginning of the cruising phase and is also the end of the climbing phase, and $t_{c f}$ is the end of the cruising phase and is also the beginning of the descent phase.

Substituting the energy expression, the performance function can be further expressed as

$$
\begin{aligned}
J= & \left.\int_{E_{i}}^{E_{c_{i}}}\left(\frac{C_{f} \dot{f}+C_{t}}{\dot{E}}\right) d E\right|_{\dot{E}>0}+\left(R-d_{u p}-d_{d n}\right) \cdot \lambda \\
& +\left.\int_{E_{c_{f}}}^{E_{f}}\left(\frac{C_{f} \dot{f}+C_{t}}{|\dot{E}|}\right) d E\right|_{\dot{E}<0}
\end{aligned}
$$

Here, $E_{n i}$ is the initial energy of the aircraft, $I_{f}$ is the final energy of the aircraft, $E_{\mathrm{li}}$ and $\mathrm{E}_{\mathrm{ke}}$ are the initial and end energy of the cruising phase, respectively. We can assume $\mathrm{E}_{c i}=\mathrm{E}_{c f}=\mathrm{E}_{c}$. It can be expressed by distance uniformly

$$
\begin{aligned}
J= & \left.\int_{E_{i}}^{E_{i c}}\left(\frac{C_{f} \dot{f}+C_{t}}{\dot{E}}\right) d E\right|_{\dot{E}>0}+\left\{R-\left[x_{u p}\left(\mathrm{E}_{c i}\right)+x_{d n}\left(\mathrm{E}_{c f}\right)\right]\right\} \cdot \lambda \\
& +\left.\int_{E_{c f}}^{E_{f}}\left(\frac{C_{f} \dot{f}+C_{t}}{|\dot{E}|}\right) d E\right|_{\dot{E}<0}
\end{aligned}
$$

Therefore, the problem of trajectory optimization is transformed into the problem of seeking the optimal control variable $V$ and thrust $T$ of the system differential Equation (3) to minimize $J$ in Equation (7).

\subsection{Solve the optimal flight trajectory}

The trajectory optimization problem has been transformed into an optimal control problem that satisfies the energy method of the aircraft model and minimizes the performance index. According to the performance indicators of the system differential Equations (3) and (7), applying the principle of minimum value, define the Hamilton function as

$$
\begin{aligned}
H= & \left.\frac{C_{f} \dot{f}+C_{t}}{\dot{E}}\right|_{\dot{E}>0}+\left.\frac{C_{f} \dot{f}+C_{t}}{|\dot{E}|}\right|_{\dot{E}<0} \\
& -\psi(E)\left(\left.\frac{V_{u p}+V_{W u p}}{\dot{E}}\right|_{\dot{E}>0}+\left.\frac{V_{d n}+V_{W u p}}{|\dot{E}|}\right|_{\dot{E}<0}\right)
\end{aligned}
$$

Where, $\psi(E)$ is co-state variable. We can get

$$
\begin{aligned}
& H_{u p}=\left.\frac{C_{f} \dot{f}+C_{t}-\lambda\left(V_{u p}+V_{W u p}\right)}{\dot{E}}\right|_{\dot{E}>0} \\
& H_{d n}=\left.\frac{C_{f} \dot{f}+C_{t}-\lambda\left(V_{d n}+V_{W d n}\right)}{|\dot{E}|}\right|_{\dot{E}>0}
\end{aligned}
$$

We can get the segmented calculation method of the optimal trajectory on vertical flight profile as follows.

The optimal trajectory of cruising phase is

$$
\lambda^{*}=\psi\left(E_{c}\right)=\operatorname{Min}_{V_{c}} \frac{C_{f} \cdot f+C_{t}}{V_{c}+V_{W}}
$$

Choosing the right flight altitude and speed can optimize direct operation costs in cruising phase. Since time is closely related to fuel consumption, the cruising plan is established by selecting the appropriate speed and flight altitude. Specific Range (SR) is defined as the distance flew per unit fuel consumption, and SR is equal to

$$
S R=\frac{V_{t}}{F F}=\frac{V_{t}}{\frac{C_{t s c}}{g} T}=\frac{V_{t}}{\frac{C_{t s c}}{g} \frac{m g}{L / D}}=\frac{V_{0} \cdot M a \cdot L / D}{\frac{C_{t s c}}{g} m g}
$$

Where, FF is fuel flow, Ma is Mach number, L/D is lift-to-drag ratio, $\mathrm{m}$ is quality, $\mathrm{g}$ is gravitational acceleration the earth, and $\mathrm{C}_{\text {tsfc }}$ is unit fuel consumption coefficient.

The optimal trajectory of climbing phase is 


$$
H_{u p}=\operatorname{Min} \frac{C_{f} \dot{f}+C_{t}-\lambda \cdot\left(V_{u p}+V_{W u p}\right)}{\dot{E}}
$$

The optimal trajectory of descent phase is

$$
H_{d n}=\operatorname{Min} \frac{C_{f} \dot{f}+C_{t}-\lambda \cdot\left(V_{d n}+V_{W d n}\right)}{|\dot{E}|}
$$

\section{Program and simulation}

We use B737-700 for the simulation in this study.

\subsection{Optimization simulation in cruising phase}

The flying altitude is constant. When the weight of the aircraft changes, the long-range cruising speed and most economical cruising speed of the aircraft are shown in Figure 1 and Figure 2.

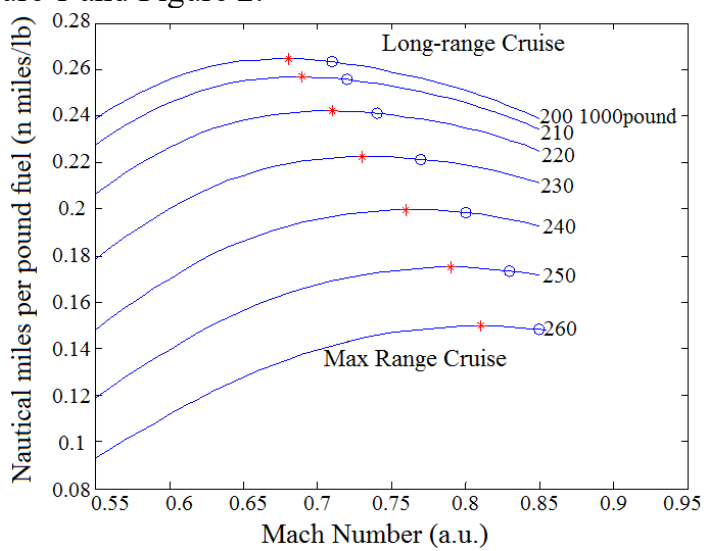

Fig. 1. Long-range cruising speeds of different qualities under $\mathrm{FL}=350$.

It can be seen from Figure 1 that the smaller weight, the greater optimal unit fuel mileage. When the unit fuel drops in a small range $(<1 \%)$, the cruising speed can be greatly increased. Therefore, the aircraft often adopts long-range cruise mode in the cruising phase.

The minimum direct operation cost corresponds to a specific Mach number, which is called economic speed.

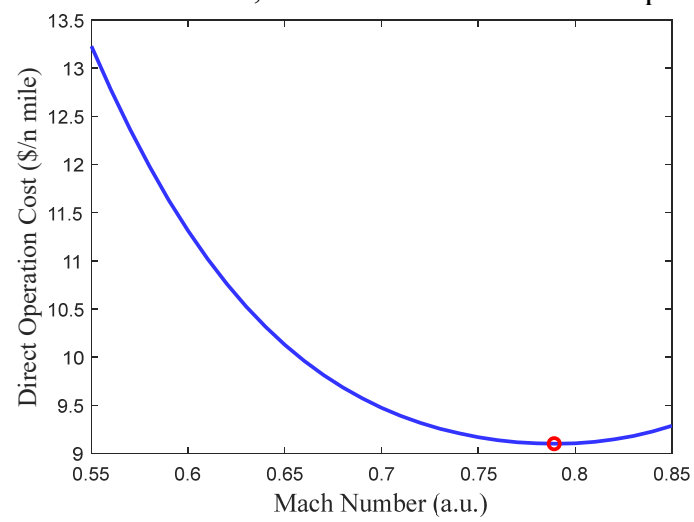

Fig. 2. Optimal cruising speed under $\mathrm{FL}=350$.

Considering the impact of time cost, we take cost index (CI), that is, time cost divided by fuel cost equals 30 . When the cruising altitude is 35000 feet, the DOC corresponding to different Mach numbers is shown in Figure 2. The most economical cruising speed is about 0.78 Mach number.

\subsection{Optimized simulation in climbing phase}

Based on the established aircraft performance database, the following three methods are compared. Method 1 is energy state approximation method and optimal control theory (ESOC) derived above and adopted in this paper. Method 2 is proportional distribution of energy (PDE), that is, flight management system proportionally distributes the increased energy of thrust to speed and altitude, referring to the general ratio of $70 \%$ for speed and $30 \%$ for altitude. Method 3 is based on the performance database of $\mathrm{B} 737-700$ (PDB). In PDB, climbing angle is

$$
\gamma=\sin ^{-1}\left(\frac{T}{W}-\frac{1}{L / D}\right)
$$

Considering the actual flight situation and air traffic control constraints, the FL100 level is the acceleration stage of the aircraft's level flight. The comparison of the three methods for climbing trajectory is shown in Figures 3,4 and 5 .

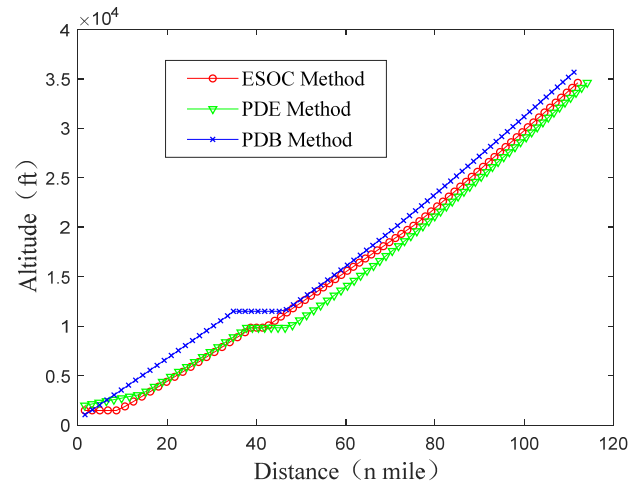

Fig. 3. The trajectory of climbing phase.

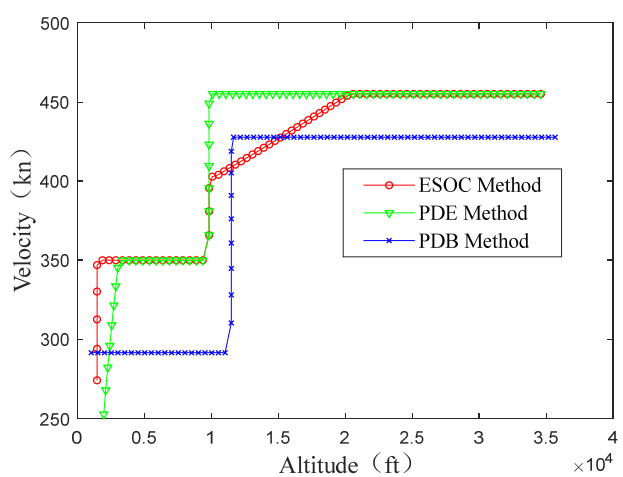

Fig. 4. Velocity at different altitudes in climbing phase.

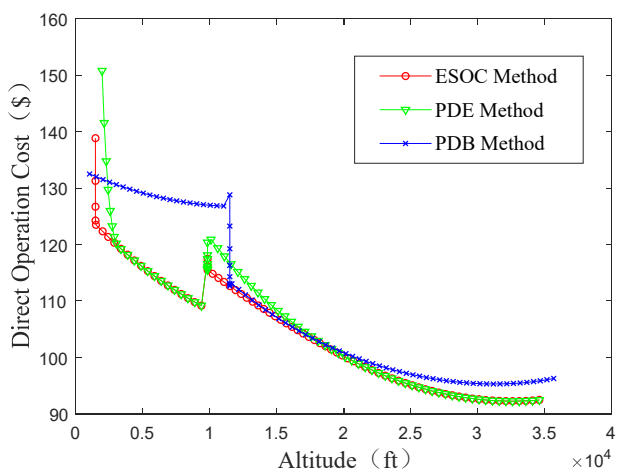

Fig. 5. Costs at different altitudes in climbing phase. 


\subsection{Optimized simulation in descent phase}

The optimization of descent phase is similar to that of the climbing phase. The difference is that the engine is in the idle state during the descent phase, and the thrust is small or negative. At the same time, considering the actual situation of the flight and air traffic control, FL100 level is the deceleration section of the aircraft's level flight. Comparing with the climbing trajectory, the three methods are shown in Figures 6,7 and 8.

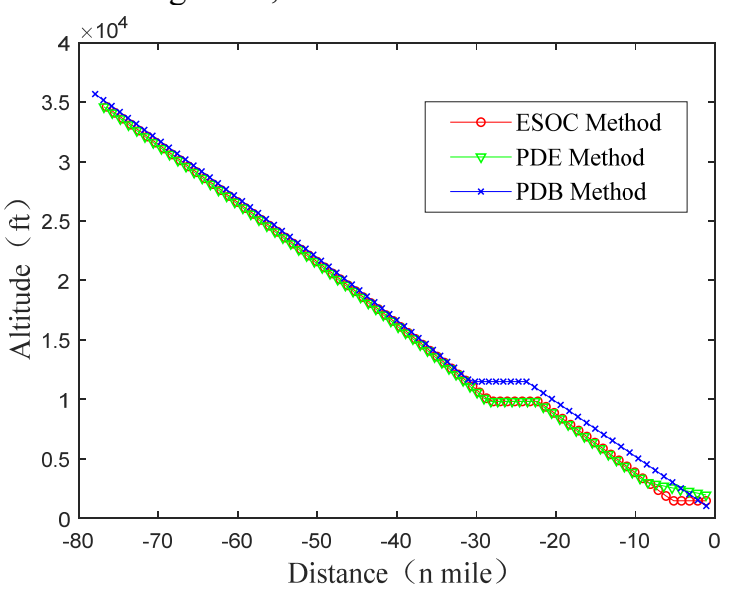

Fig. 6. The trajectory in descent phase.

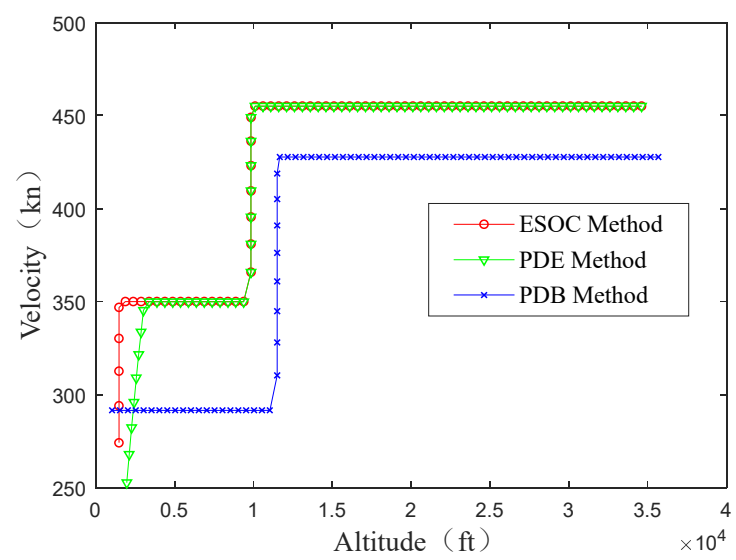

Fig. 7. Velocity at different altitudes in descent phase.

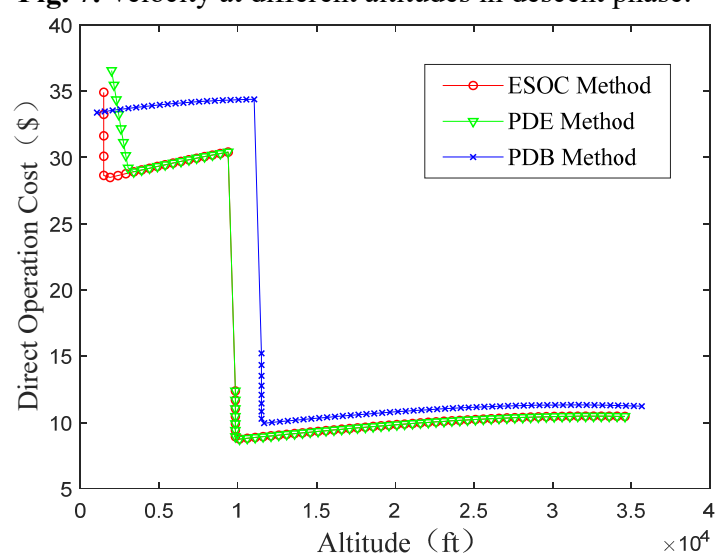

Fig. 8. Costs at different altitudes in descent phase.

\subsection{Flight cost analysis}

Taking the example from Shanghai to Beijing, long-range Mach number 0.74 is used for the comparison of cruising phase according to PDB.
Table 1. The flight cost calculated with the three methods.

\begin{tabular}{|c|c|c|c|c|}
\hline $\begin{array}{c}\text { Velocity } \\
\text { type }\end{array}$ & $\begin{array}{c}\text { Climbing } \\
\text { cost (\$) }\end{array}$ & $\begin{array}{c}\text { Descent } \\
\text { cost (\$) }\end{array}$ & $\begin{array}{c}\text { Cruising } \\
\text { cost (\$) }\end{array}$ & $\begin{array}{c}\text { Total } \\
\text { cost (\$) }\end{array}$ \\
\hline ESOC & 1650.2 & 663.2 & 3149.3 & 5462.6 \\
\hline PDE & 1689.1 & 674.3 & 3200.5 & 5563.9 \\
\hline PDB & 1677.8 & 780.0 & 3200.5 & 5658.3 \\
\hline
\end{tabular}

It can be seen from the simulation results that the total flight cost of ESOC method is reduced by $1.88 \%$ and $3.46 \%$ respectively compared with the other two methods.

\section{Conclusions}

This paper establishes a performance optimization model on vertical flight profile of the aircraft, optimizes and simulates trajectory of the aircraft in climbing, cruising, and descent phases. Comparing with PDE and PDB methods, the total flight cost of ESOC method is reduced by $1.88 \%$ and $3.46 \%$, respectively. The optimization effect of the aircraft flight cost is obvious and it has a certain practical value.

We will further conduct more systematic and accurate modelling and analysis of aircraft atmospheric environment models. The thrust model of aircraft will be also improved.

\section{References}

1. A. Franco, D. Rivas, Optimization of Multiphase Aircraft Trajectories Using Hybrid Optimal Control [J]. Journal of Guidance Control and Dynamics, 38 (3): 452-167(2015).

2. M. Soler, A. Olivares, E. Staffetti, Multiphase Optimal Control Framework for Commercial Aircraft Four-Dimensional Flight-Planning Problems [J]. Journal of Aircraft, 52 (1): 274-286(2015).

3. S. G. Park, P. Dutta and P. K. Menon, Optimal Trajectory Option Sets for In-Flight Climb-Descend Trajectory Negotiations [J]. 17th AIAA Aviation Technology, Integration, and Operations Conference, 3432: 1-17 (2017).

4. S. G. Park, J. B. Clarke, Optimal Control Based Vertical Trajectory Determination for Continuous Descent Arrival Procedures [J]. Journal of Aircraft, 52 (5): 1469-1480 (2015).

5. O. S. Meric, O. Turan, Evaluation of Aircraft Descent Profile [J]. Energy Procedia, 95: 308-313(2016).

6. K. N. Lv, Y. Nan, Climb Trajectory Optimization of Aircraft based on Adaptive Genetic Algorithm [J].Computer Simulation, 34(1):66-69 (2017). 